\title{
Effect of Rifapentine and Rifampicin on Anti-Tuberculosis
}

\author{
Weijia Lin1, Yaping Zhang', Zhi Liu², Ping Feng' ${ }^{1}$, Zhihua Zhang1, Xiulong Zhang1 \\ ${ }^{1}$ Department of Respiratory Medicine, The First Affiliated Hospital of Hebei North University, Zhangjiakou, China \\ ${ }^{2}$ Department of Orthopedic Surgery, The First Affiliated Hospital of Hebei North University, Zhangjiakou, China \\ Email:1wj1987lu@163.com
}

How to cite this paper: Lin, W.J., Zhang, Y.P., Liu, Z., Feng, P., Zhang, Z.H. and Zhang, X.L. (2021) Effect of Rifapentine and Rifampicin on Anti-Tuberculosis. Journal of Tuberculosis Research, 9, 205-210. https://doi.org/10.4236/jtr.2021.93019

Received: August 12, 2021

Accepted: September 12, 2021

Published: September 15, 2021

Copyright $\odot 2021$ by author(s) and Scientific Research Publishing Inc. This work is licensed under the Creative Commons Attribution International License (CC BY 4.0).

http://creativecommons.org/licenses/by/4.0/

\begin{abstract}
Objective: To investigate the clinical effect of rifapentine and rifampicin in the treatment of pulmonary tuberculosis. Methods: Seventy-two cases of patients with initial treatment of pulmonary tuberculosis who attended the First Hospital Affiliated to Hebei North University from February 2017 to August 2019 were selected. They were randomly divided into observation group and control group, with 36 cases in each group. The observation group was treated with isoniazid + rifapentine + ethambutol, while the control group was treated with isoniazid + rifampicin + ethambutol. The symptom relief, image absorption and adverse reactions were compared between the two groups. Results: The rate of symptom relief was $86.11 \%$ in the observation group and $94.44 \%$ in the control group, $\mathrm{P}<0.05$, which was statistically significant. Rifampin was more helpful than rifapentine in relieving clinical symptoms. The lesion absorption rate was $77.79 \%$ in the observation group and $88.89 \%$ in the control group, $\mathrm{P}<0.05$, and the difference was statistically significant. Rifampin was more beneficial to the absorption of TB lesions than rifapentine. The incidence of adverse reactions in the observation group was $16.67 \%$ much lower than that in the control group, which was $38.89 \%$, indicating that the adverse reactions of rifapentine were less. Conclusion: Rifampicin is superior to rifapentine in clinical symptom relief and lesion absorption, but the incidence of adverse reactions is high.
\end{abstract}

\section{Keywords}

Rifampicin, Rifapentine, Pulmonary Tuberculosis

\section{Introduction}

Tuberculosis is an infectious disease caused by Mycobacterium tuberculosis infection in the lungs [1], it has been on the rise in recent years, and the incidence rate is increasing year by year, which seriously threatens the health and mental health 
of the patients. The current situation of pulmonary tuberculosis remains critical and remains a public health problem of key concern to society [2]. Rifampicin [3] [4] is a widely used antibiotic clinical practice. It is the first choice for the treatment of pulmonary tuberculosis. Its clinical pharmacological mechanism mainly refers to blocking the activity of RNA polymerase, blocking the link between RNA polymerase and deoxyribonucleic acid (DNA), then blocking RNA transcription and inhibiting cell proliferation, so as to kill Mycobacterium tuberculosis. However, the adverse reactions of rifampicin are large, especially gastrointestinal reactions, liver dysfunction, gastritis, liver diseases and the expansion of adverse reactions in elderly and frail patients. Rifapentine [5] [6] is a rifampicin derivative, and its clinical pharmacological mechanism is highly consistent with rifampicin. The half-life of the drug is prolonged. Adverse reactions are less. Studies have shown that rifapentine is effective in the treatment of pulmonary tuberculosis [7]. It has little effect on liver function. The aim of this study was to investigate the efficacy of rifapentine and rifampicin on symptom relief, lesion absorption and adverse effects in patients with pulmonary tuberculosis and to assess the efficacy of both.

\section{Materials and Methods}

\subsection{Materials}

A total of 72 patients with initial treatment of pulmonary tuberculosis who attended the First Hospital of Hebei North University from February 2017 to August 2019 were selected. The cases were randomly divided into observation group and control group, with 36 cases in each group. The observation group was treated with isoniazid + rifapentine + ethambutol, while the control group was treated with isoniazid + rifampicin + ethambutol. Observation group (rifapentine): 19 males and 17 females, aged 19 - 74 years, with an average age of (50.28 \pm 15.64$)$. In the control group (rifampicin), there were 15 males and 21 females, aged $23-75$ years, with an average age of $(51.86 \pm 14.19)$. There was no significant difference in the general data between the two groups $(\mathrm{P}>0.05)$.

\subsection{Inclusion and Exclusion Criteria}

Inclusion criteria: 1) Conforming to the diagnostic criteria of the Chinese Medical Association 2004 "Clinical Diagnosis and Treatment Guidelines for Tuberculosis"; 2) All patients were first diagnosed; 3) Age $\leq 75$ years; 4) The patients had informed consent and good compliance, and cooperated with follow-up.

Exclusion criteria: 1) Patients with arrhythmia, heart failure, liver and kidney dysfunction and malignant tumor; 2) Patients who have relapsed or have been treated with antituberculosis; 3) $75>$ Age $\leq 18$ years; 4) Poor compliance, unable to cooperate with follow-up.

\subsection{Grouping and Administration}

\subsubsection{Grouping}

Observation group: Isoniazid + Rifapentine + Ethambutol for 3 months.

Control group: Isoniazid + Rifampicin + Ethambutol for 3 months. 


\subsubsection{Drug Administration Method}

One single administration on an empty stomach in the morning:

Isoniazid $0.3 \mathrm{~g} / \mathrm{d}$, Rifapentine $0.6 \mathrm{~g}$ 2/w, Rifampicin $0.45 \mathrm{~g} / \mathrm{d}$, Ethambutol 0.75 g/d.

After three meals:

Glucuronolactone $200 \mathrm{mg} / \mathrm{once}, 3 / \mathrm{d}$.

The liver and kidney functions were rechecked every 2 weeks, and the chest CT was rechecked every 4 weeks.

\subsection{Observation Index}

The symptom relief (Effective: within 2 - 12 weeks after treatment, the symptoms of low fever, night sweats, fatigue and cough were significantly relieved or even disappeared. Ineffective: symptoms such as low fever, night sweat, fatigue and cough did not improve within 2 - 12 weeks after treatment.), image absorption and adverse reactions of the two groups were compared.

\subsection{Statistical Method [8]}

Spss20.0 statistical software was used to process data. The measurement data is expressed in $\bar{X} \pm \mathrm{s}$, $\mathrm{t}$-test is performed. The count data is expressed in $\%, \chi^{2}$ is performed. $\mathrm{P}<0.05$ means the difference is statistically significant.

\section{Results}

\subsection{Comparison of Remission of Tuberculosis Symptoms}

The relief of symptoms such as low fever, night sweats, fatigue and cough were compared between the two groups. A total of 31 patients in the observation group felt ideal symptom relief, and the relief rate was $86.11 \%$. A total of 33 patients in the control group felt ideal symptom relief, and the total remission rate was $94.44 \%$, which was higher than $8.33 \%$ in the observation group. $\mathrm{P}=0.032<0.05$, the difference was statistically significant (Table 1 ). It shows that rifampicin is more helpful to alleviate symptoms than rifapentine.

\subsection{Image Absorption}

The absorption of chest CT tuberculosis was compared between the two groups. In the observation group, 28 patients were absorbed by tuberculosis foci, 8 cases

Table 1. Symptom relief in observation group and control group.

\begin{tabular}{cccccccc}
\hline Group & $\begin{array}{c}\text { Total } \\
\text { cases }\end{array}$ & $\begin{array}{c}\text { Remission } \\
\text { cases }\end{array}$ & 2 week & 4 week & 8 week & 12 week & $\begin{array}{c}\text { Total } \\
\text { remission rate }\end{array}$ \\
\hline $\begin{array}{c}\text { Observation group } \\
\text { Control group }\end{array}$ & 36 & 31 & 9 & 8 & 10 & 4 & $86.11 \%$ \\
$\chi^{2}$ & 36 & 34 & 12 & 11 & 9 & 2 & $94.44 \%$ \\
$\mathrm{P}$ & & & & & & & 8.785 \\
\hline
\end{tabular}


were not absorbed, and the absorption rate was $77.79 \%$. In the control group, 32 patients were absorbed by the tuberculosis foci, 4 of them were not absorbed, and the absorption rate was $88.89 \% . \chi^{2}=18.750, \mathrm{P}=0.044<0.05$, the difference was statistically significant (Table 2). Rifampicin than Rifapentin is more conducive to the absorption of tuberculosis.

\subsection{Adverse Reactions [9] [10]}

Comparing the adverse reactions in the two groups, a total of 20 patients had adverse reactions accounting for $27.78 \%$, including 8 cases of nausea, 4 cases of loss of appetite, 7 cases of elevated transaminases and 1 case of skin rash, with incidence rates of $11.11 \%, 5.56 \%, 9.72 \%$ and $1.39 \%$, respectively. In the rifapentine group, there were 6 patients with adverse reactions, including 2 cases of nausea, 1 case of loss of appetite, 2 cases of elevated transaminases and 1 case of rash, with incidence rates of $5.55 \%, 2.78 \%, 5.55 \%$ and $2.78 \%$, respectively. A total of 13 patients in the rifampicin group had adverse reactions accounting for $38.89 \%$, including 6 cases of nausea, 3 cases of loss of appetite, 5 cases of elevated transaminases, and 0 cases of rash, with incidence rates of $16.67 \%, 8.33 \%, 13.89 \%$, and $0 \%$, respectively (see Table 3 ). The incidence of adverse reactions was higher in the rifampicin group than in the rifapentine group.

\section{Discussion}

In recent years, with the globalization of the economy, the widening gap between rich and poor and the increase of rural-urban population mobility, the incidence of tuberculosis is on the rise, which seriously threatens people's physical and mental health. Our medical treatment radiates to Hebei, Shanxi and Inner Mongolia provinces, where the mining and metal smelting industries are more developed and the proportion of people working in the mining industry is large, leading to a high proportion of silicosis, which is prone to secondary tuberculosis, resulting

Table 2. Absorption of chest CT lesions of the two groups.

\begin{tabular}{ccccc}
\hline Group & $\mathbf{n}$ & Absorbed & Unabsorbed & Absorption rate \\
\hline Observation group & 36 & 28 & 8 & $77.79 \%$ \\
Control group & 36 & 32 & 4 & $88.89 \%$ \\
$\chi^{2}$ & & & 18.750 \\
$\mathrm{P}$ & & & 0.044 \\
\hline
\end{tabular}

Table 3. Adverse reactions of the two groups.

\begin{tabular}{ccccccc}
\hline Group & $\mathbf{n}$ & Nausea & Anorexia & $\begin{array}{c}\text { Elevated } \\
\text { transaminase }\end{array}$ & Rash & $\begin{array}{c}\text { Incidence } \\
\text { rate (\%) }\end{array}$ \\
\hline Observation group & 36 & $2(5.55)$ & $1(2.78)$ & $2(5.55)$ & $1(2.78)$ & $16.67 \%$ \\
Control group & 36 & $6(16.67)$ & $3(8.33)$ & $5(13.89)$ & $0(0)$ & $38.89 \%$ \\
$\quad$ Total & 72 & $8(11.11)$ & $4(5.56)$ & $7(9.72)$ & $1(1.39)$ & $27.78 \%$ \\
\hline
\end{tabular}


in an increased incidence of tuberculosis [11] [12]. The treatment of pulmonary tuberculosis is based on the principle of sufficient quantity, whole process, regularity, appropriate amount and combination. But the treatment process is long, the effect is slow, the side effects are large, it is easy to relapse and produce drug resistance [13] [14], which leads to high psychological pressure, lack of treatment confidence and increased family economic burden, which is not conducive to family harmony and social environment stability. Searching for safer and more efficient anti tuberculosis drugs has important clinical research value.

Rifampicin is the first choice drug for antituberculosis. Its clinical pharmacological mechanism mainly refers to blocking the activity of ribonucleic acid (RNA) polymerase. Rifapentine is a derivative of rifampin, and its clinical pharmacological mechanism is highly consistent with that of rifampin, and some studies have shown that the clinical effect of rifapentine is stronger than that of rifampin, and its antimicrobial activity in the body is higher, and it can reach the peak blood concentration rapidly with a longer half-life. This study showed that the symptom relief rate of $86.11 \%$ in the rifapentine group was lower than that of $94.44 \%$ in the rifampicin group, $\mathrm{P}<0.05$, indicating that rifapentine was less effective than rifampicin in terms of clinical symptom relief and absorption of tuberculosis lesions. The rate of lesion absorption in the rifapentine group was $77.79 \%$, which was lower than that in the rifampin group (88.89\%). The incidence of adverse reactions in the rifapentine group was $16.67 \%$ much lower than that in the control group (38.89\%), indicating that rifapentine had fewer adverse reactions. Both rifapentine and rifampin could play a good role in clinical symptom relief and lesion absorption, and rifampin was more effective with a high incidence of adverse reactions, but no serious adverse reactions were found to interfere with the treatment plan. The research results fail to show that rifapentine takes advantage of rifampicin over rifampicin depending on its high blood concentration and long half-life. It is considered that it is related to the frequency of taking rifampicin. Rifampicin takes medicine once a day, the same as isoniazid and ethambutol, which is not easy to be omitted. However, rifapentine takes medicine twice a week and needs to be taken at an interval of 2 - 3 days, especially in elderly patients with low educational level, with poor memory, Poor compliance, is easy to miss drugs, resulting in unstable blood drug concentration and affecting the antibacterial effect. At the same time, because some patients were thin, the reduction of rifapentine was $0.45 \mathrm{~g}$, which weakened the antibacterial strength. Therefore, the antituberculosis effect of rifapentine in this study is less than that of rifampicin. Only 72 cases were included in this study. The sample size is small and large sample clinical research is needed. The results of this study may have limitations and are expected to be confirmed by big data research.

\section{Conflicts of Interest}

The authors declare no conflicts of interest regarding the publication of this paper. 


\section{References}

[1] National Health Commission of the People's Republic of China (2017) WS 288-2017 Diagnosis for Pulmonary Tuberculosis Standards.

[2] Hao, W., Shang, X.S. and Liu, C.G. (2019) Comparison Efficacy of Moxifloxacin and Levofloxacin in the Treatment of Patients with Multidrug Resistant Pulmonary Tuberculosis. Medical Innovation of China, 16, 62-65.

[3] Hao, W., Guo, G.L. and Liu, C.G. (2019) Effect of Isoniazid p-Aminosalicylate Combined with Rifapentine in the Treatment of Refractory Pulmonary Tuberculosis. Journal of Clinical Rational Drug Use, 12, 56-57.

[4] Lian, H.M., Yang, Z.F. and Yuan, H.F. (2019) Comparison of Clinical Effects of Rifapentine and Rifampicin against Tuberculosis Infection Program in the Treatment of Tuberculosis and Its Effect on Levels of Inflammatory Cytokines. Clinical Research and Practice, 4, 50-51.

[5] Zhang, Q. (2021) Efficacy of Rifapentine Capsule Combined with Standard Anti Tuberculosis Drugs in the Treatment of Elderly Patients with Pulmonary Tuberculosis. Medical Equipment, 34, 95-96.

[6] Zhou, W.L. (2021) Efficacy and Safety of Rifapentine in the Treatment of Pulmonary Tuberculosis. Chinese Journal of Modern Drug Application, 15, 167-169.

[7] Guo, Y.Y. (2021) Effect of Rifapentine and Rifampicin in the Treatment of Pulmonary Tuberculosis and Its Effect on Liver Function. Guide of China Medicine, 19, 100101.

[8] Li, H. (2021) Clinical Efficacy and Safety of Moxifloxacin and Levofloxacin in the Treatment of Patients with Multi Drug Resistant Pulmonary Tuberculosis. Chinese Journal of Modern Drug Application, 15, 158-159.

[9] Tian, Y.L. (2016) Efficacy and Safety Evaluation of Rifapentine in the Treatment of Pulmonary Tuberculosis. Journal of Clinical Medical Literature, 3, 94-96.

[10] Guan, J. (2008) Comparison of Adverse Reactions and Efficacy of Rifampicin and Rifapentine in Antituberculosis. China Medical Herald, No. 7, 65-66.

[11] Song, Y. (2018) Clinical Analysis of Silicosis Complicated with Pulmonary Tuberculosis. Systems Medicine, 3, 38-40.

[12] Wang, R.F. (2021) Clinical Characteristics of Senile Pneumoconiosis. Chinese Journal of Industrial Medicine, 34, 176-178.

[13] Bai, S.J. (2021) Comparison of Clinical Efficacy of Levofloxacin and Moxifloxacin in the Treatment of Multi Drug Resistant Pulmonary Tuberculosis. Chinese Journal of Modern Drug Application, 15, 170-172.

[14] Lv, Y.Q. and Shi, L. (2021) Analysis of the Clinical Effects and Adverse Reactions of Moxifloxacin Combined with Capreomycin in the Treatment of Multidrug-Resistant Pulmonary Tuberculosis. China Practical Medicine, 16, 106-108. 